\title{
ADIÇÃO DE CATALISADOR À MISTURA DE CARVÕES NO PROCESSO DE COQUERIA DA CSN*
}

\author{
Sidiney Nascimento Silva ${ }^{1}$ \\ Agenor Medrado da Silva² \\ Andre Luiz Azevedo ${ }^{3}$ \\ Natalie de Lacerda Citeli Silveira ${ }^{4}$ \\ Carlos Alberto de Oliveira Faria ${ }^{5}$ \\ Alexandre Lucena Avelar ${ }^{6}$
}

\section{Resumo}

O trabalho descreve os principais resultados dos testes industriais realizados na Usina Presidente Vargas, CSN, Brasil, envolvendo a adição de um catalisador à mistura de carvões, em termos da qualidade do coque e do processo da coqueria. O catalisador em questão é o produto Thermact, que possui partículas nanométricas em sua composição, fornecido pela empresa Abhitech Energycon Limited, Índia. O catalisador foi dosado e adicionado à mistura de carvões via correia transportadora, na proporção, em peso, de $1 \mathrm{~kg}$ de catalisador para $4.000 \mathrm{~kg}$ da mistura de carvões. Os resultados dos testes realizados nas Baterias \# 1, 4A, 4B e 5 com a adição de um catalisador à mistura de carvões, sinalizaram a viabilidade técnica e financeira da sua aplicação no processo da coqueria. Os principais benefícios decorrentes da adição do catalisador foram: i) aumento potencial da capacidade de produção de coque ao redor de $10 \%$ ou, alternativamente, melhoria da qualidade do coque - aumento de $1,4 \%$ do valor médio do índice de resistência mecânica a frio do coque, DI150/15, mantendo-se o tempo total padronizado do processo de coqueificação, visando à preservação das Baterias, ou ainda, a utilização de uma mistura de carvões de menor custo, sem prejuízos ao atendimento à especificação do coque para os Altos-Fornos; b) aumento da geração e redução do consumo de gás de coqueria, GCO; iii) aumento na produção de BTX (Benzeno, Tolueno, Xileno) e redução da produção de alcatrão na Planta de Carboquímicos; iv) limpeza do sistema "PROven", "goosenecks", tubos de ascensão e paredes dos fornos, sinalizando a sua viabilidade técnica e econômica.

Palavras-chave: Catalisador; Carvão; Coque; Coqueria.

\begin{abstract}
ADDITION OF CATALYST TO THE COAL BLEND AT CSN COKE OVEN PLANT
\section{Abstract}

This paper describes the main results of industrial trials carried out at Usina Presidente Vargas, Companhia Siderúrgica Nacional, CSN, Brazil, involving the addition of catalyst to the coal blend in terms of coke quality and coking process. The so-called catalyst, that has particles with nanometric dimensions in its composition, is a product supplied by Abhitech Energycon Limited, from India. The catalyst was dosed and added to the coal blend through belt conveyor, in the proportion, in mass, of $1 \mathrm{~kg}$ of catalyst to $4.000 \mathrm{~kg}$ of coal blend. The results of industrial trials carried out at Coke Bateries \# 1, 4A, 4B and 5, with the addition of the catalyst to the coal blend, have showed technical and financial feasibility of its application at coking process. The main benefits from the catalyst addition to the coal blend were the following: a) potential increasing of production capacity around $10 \%$ or, alternatively, improvement of coke quality - increasing in $1,4 \%$ of the cold mechanical resistance index, DI $150 / 15$, average value, by keeping the standard total time of coking process in order to preserve the coke Bateries, or even the utilization of a cheaper coal blend, with lower cost coals, without losses to the specified coke quality for the blast furnaces; b) increasing of generation and reduction of consumption of coke oven gas, COG; $c$ ) increasing of BTX production (benzene, toluene and xylene) and reduction of tar production from by-products plant; and d) cleaning up of "PROven" system, goosenecks, ascension pipes and oven sidewalls, indicating its technical and economic feasibility.
\end{abstract}

Keywords: Catalyst; Coal; Coke; Coke oven plant.

1 Engenheiro Civil, Doutor em Ciência e Engenharia de Materiais, Gerente de Processos da Metalurgia, Companhia Siderúrgica Nacional (CSN), Volta Redonda, RJ, Brasil.

2 Eng. Metalúrgico, Doutor em Eng. Metalúrgica, Engenheiro Especialista, CSN, Volta Redonda, RJ, Brasil.

3 Técnico de Metalurgia, Técnico especialista, Gerência de Coqueria, CSN, Volta Redonda, RJ, Brasil.

4 Engenheira Metalúrgica, Engenheira Jr., Gerência de Coqueria, CSN, Volta Redonda, RJ, Brasil.

5 Técnico de Metalurgia, Supervisor de Controle Térmico das Baterias, Gerência de Coqueria, CSN, Volta Redonda, RJ, Brasil.

6 Líder de Controle Térmico das Baterias, Gerência de Coqueria, CSN, Volta Redonda, RJ, Brasil.

* Contribuição técnica ao $44^{\circ}$ Seminário de Redução de Minério de Ferro e Matérias-primas, $15^{\circ}$ Simpósio Brasileiro de Minério de Ferro e $2^{\circ}$ Simpósio Brasileiro de Aglomeração de Minério de Ferro, 15 a 18 de setembro de 2014, Belo Horizonte, MG, Brasil. 


\section{INTRODUÇÃO}

O catalisador THERMACT é um produto fornecido pela empresa Abhitech Energycon Limited, Índia. As principais características do referido catalisador são [1]:

i) Produto granulado, termoativo e multifuncional.

ii) Possui partículas com dimensões nanométricas em sua composição.

iii) Todos os elementos presentes em sua composição química são de natureza orgânica.

iv) Ativação a partir de $525^{\circ} \mathrm{C}$.

De acordo com o fabricante, o catalisador THERMACT interage com os carvões metalúrgicos, promovendo as reações intermediárias do carbono do carvão com as moléculas de água contidas em sua estrutura - umidade intrínseca. A molécula de água é dissociada, liberando os átomos de oxigênio e hidrogênio. Os átomos livres de hidrogênio reagem então com os átomos de carbono, levando à formação do gás metano, $\mathrm{CH}_{4}$, que após combustão, gera calor, Figura 1.

$$
\begin{aligned}
& \mathrm{C}+2 \mathrm{H}_{2} \mathrm{O} \stackrel{\text { THERMACT }}{\longrightarrow}\left(\mathrm{C}+2 \mathrm{H}_{2}\right)+2 \mathrm{O} \text { (oxigênio dissociado) }
\end{aligned}
$$



Figura 1 - Interação do catalisador THERMACT com os carvões metalúrgicos.

Com o objetivo de avaliar o efeito do catalisador THERMACT sobre a qualidade do coque e sobre o processo da coqueria, a CSN e a Abhitech Energycon decidiram planejar e realizar testes industriais nas Baterias de Coque da Usina Presidente Vargas, UPV, envolvendo a adição do referido catalisador à mistura de carvões de coqueria.

O catalisador foi dosado e adicionado à mistura de carvões via correia transportadora, na proporção, em peso, de $1 \mathrm{~kg}$ de catalisador para $4.000 \mathrm{~kg}$ da mistura de carvões.

Os principais benefícios potenciais decorrentes da adição do catalisador THERMACT à mistura de carvões de coqueria são:

- Redução do tempo necessário para atingir a temperatura máxima de coqueificação da mistura de carvões.

- Geração adicional de calor, resultando em um melhor perfil de temperatura dos fornos de coqueificação e, por conseguinte, menor consumo de gás de coqueria.

- Redução do tempo total de coqueificação da mistura de carvões, sem efeito colateral negativo sobre a qualidade do coque.

- Aumento da capacidade de produção de coque, em função da redução do tempo total de coqueificação ou, alternativamente, aumento dos valores de DI e CRI, índice de resistência mecânica a frio e índice de reatividade, respectivamente, do coque produzido.

- Aumento na geração do gás de coqueria decorrente da otimização do processo.

- Limpeza do sistema "PROven", "goosenecks", tubos de ascensão e paredes dos fornos. 


\section{TESTES INDUSTRIAIS}

Os testes industriais realizados com a adição do catalisador THERMACT à mistura de carvões no processo de coqueria da CSN totalizaram o consumo de $18.500 \mathrm{~kg}$ do referido catalisador.

Adicionou-se o catalisador à mistura de carvões através da correia transportadora da rota de abastecimento dos fornos das Baterias de coque. No período de 23 de Outubro a 08 de Novembro de 2013, os testes com o catalisador foram restritos à Bateria \# 1, composta de 75 fornos de $4,5 \mathrm{~m}$ de altura - bateria baixa. A dosagem foi realizada na proporção de $1 \mathrm{~kg}$ de catalisador para $4.000 \mathrm{~kg}$ de mistura de carvões, com auxílio de um dosador vibratório dotado de um alternador de frequência. Um silo com capacidade de $500 \mathrm{~kg}$ foi montado sobre a correia transportadora para receber manualmente o catalisador conforme ilustrado na Figura 2.
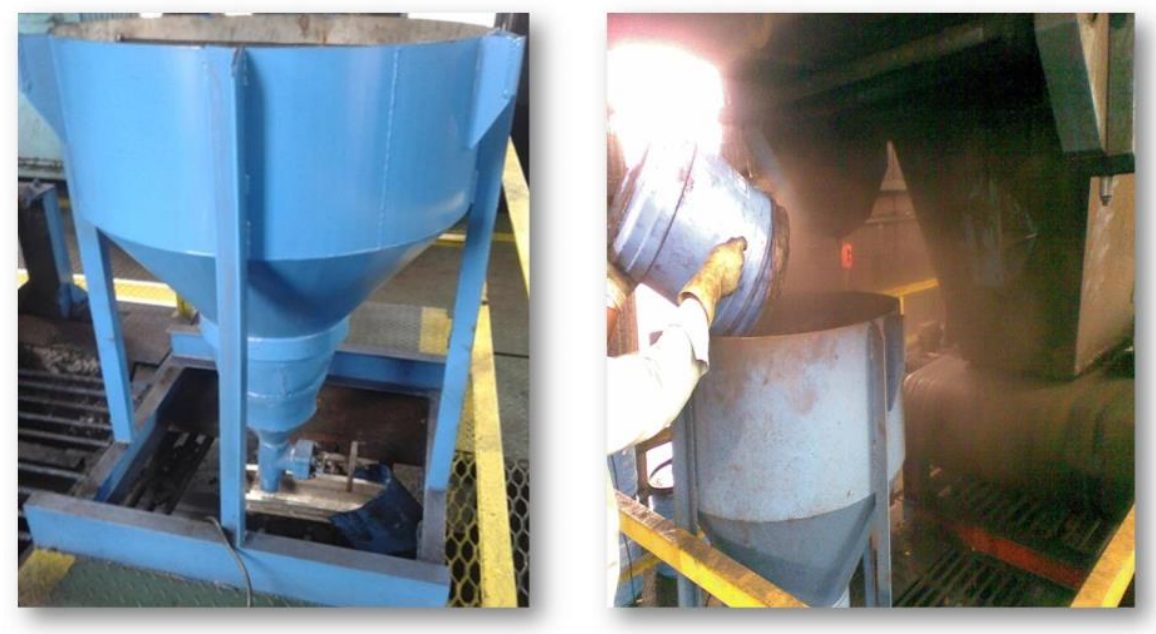

Figura 2 - Silo com dosador vibratório e abastecimento manual do silo com o catalisador.

No período de 2 a 22 de Novembro de 2013, o catalisador foi adicionado à mistura de carvões que abastece as Baterias \# 4A, 4B e 5. Essas Baterias totalizam 105 fornos, com $6 \mathrm{~m}$ de altura - baterias altas. Todavia no período de testes, apenas 60 fornos estavam em operação, uma vez que os demais fornos, 45, encontravam-se em reparo.

No período de 02 a 08 de Novembro de 2013, todos os fornos em operação das Baterias receberam a adição do catalisador THERMACT.

Testes adicionais para avaliar a ação do catalisador sobre a eficiência da limpeza do sistema PROven - Pressure Regulated Oven, Figura 3, também foram realizados no período de 23 a 29 de Novembro de 2013, em 9 (nove) fornos especialmente selecionados - Fornos \# 96, 104, 123, 153, 154, 155, 156, 157 e 162. Durante o mesmo período, foram selecionados os Fornos \# 123 e 162 para efeito da avaliação da ação do catalisador sobre a eficiência da limpeza do "fixcup" e "rolha" - material depositado e aderido no interior do PROven, através de inspeção visual. 

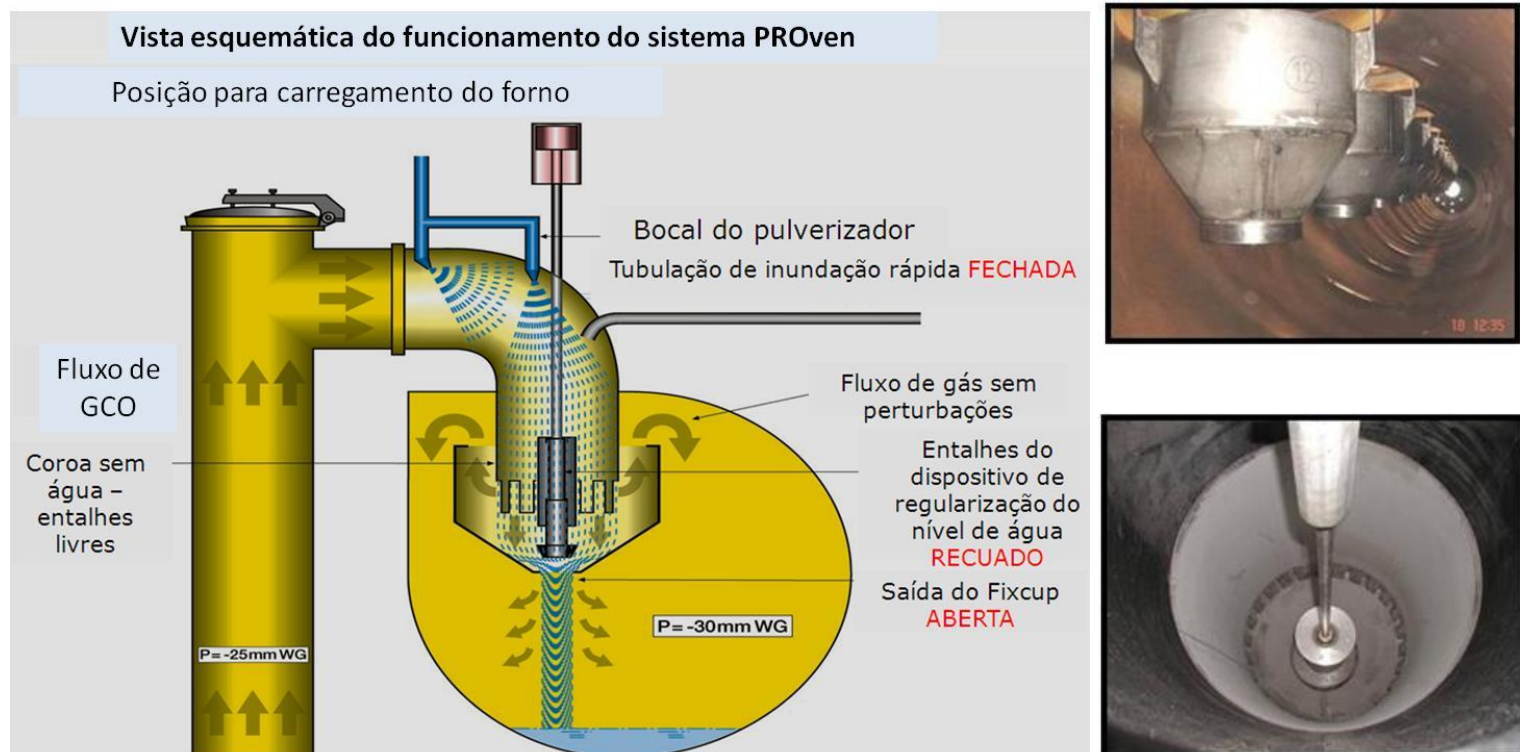

Figura 3 - Funcionamento esquemático do sistema "PROven".

\section{RESULTADOS DOS TESTES INDUTRIAIS}

\subsection{Bateria de Fornos \# 1}

\subsubsection{Qualidade do coque}

Inicialmente, observou-se uma redução do tempo necessário para atingir a temperatura máxima de coqueificação da mistura de carvões em cerca de 2 (duas) horas, viabilizando, tecnicamente, uma redução do tempo total do processo de coqueificação de 17 para 15 horas. Isso significa um aumento potencial da capacidade de produção ao redor de $10 \%$. Todavia, em função das condições particulares das Baterias de Fornos de Coque da CSN, com elevado tempo em operação, ao redor de 24 anos, decidiu-se não aumentar a sua taxa operacional, mantendo-se o tempo total padronizado do processo de coqueificação, de 17 horas, visando à sua preservação. Como resultado, verificou-se um aumento de $1,4 \%$ do valor médio do índice de resistência mecânica a frio do coque, DI 150/15. A Figura 4 apresenta uma comparação da qualidade do coque coletado na Rampa \# 1, não estabilizado, em termos do índice de resistência mecânica a frio, DI 150/15, com e sem a adição do catalisador, tomando-se como referência os Fornos \# 37, 42, 47 e 62 da Bateria \# 1, bateria baixa.

Esse efeito positivo do catalisador sobre a qualidade do coque possibilita, alternativamente, a utilização de carvões de menor custo na mistura, sem prejuízos ao atendimento à especificação do coque para os Altos-Fornos - valor mínimo do DI de $85 \%$, coque estabilizado, no caso particular da CSN. Isso significa uma redução dos custos com a aquisição dos carvões de coqueria. 

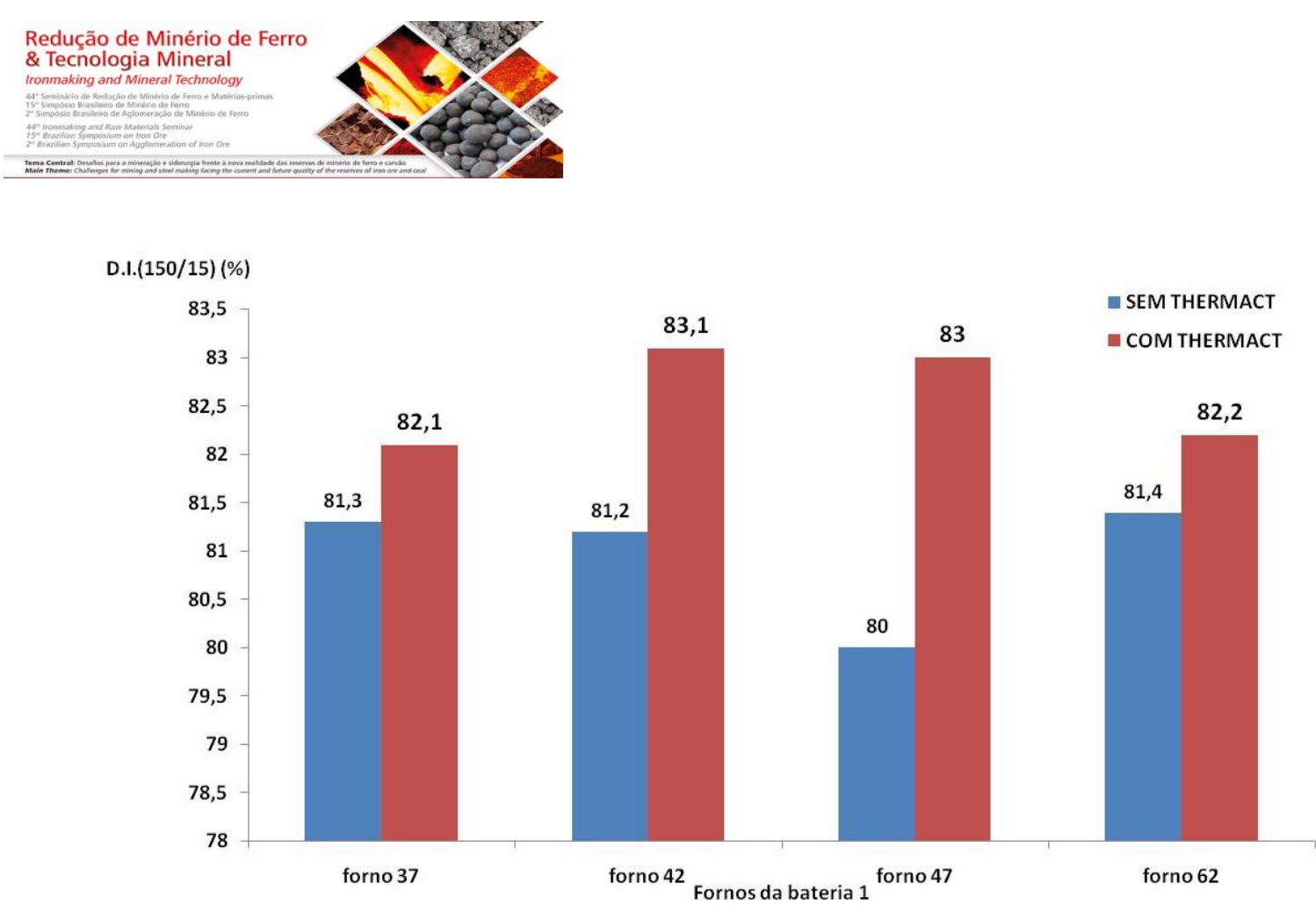

Figura 4 - Comparação dos resultados de qualidade do coque , DI ${ }^{150 / 15}$, coletado na Rampa \# 1, não estabilizado, dos Fornos \# 37, 42, 47 e 62 da Bateria \# 1.

\subsubsection{Geração e consumo de gás de coqueria (GCO)}

Durante o período de testes com a adição do catalisador na Bateria \# 1, houve um aumento de cerca de $11 \%$ na geração de gás de coqueria, GCO, de 1.300 para $1.445 \mathrm{dam}^{3} / \mathrm{dia}$, decorrente da otimização do processo. Por outro lado, em função do melhor perfil de temperatura dos fornos, ocorreu, simultaneamente, uma redução de $5 \%$ do consumo de GCO no processo da coqueria, de 0,53 para 0,48 dam³/tonelada de coque. Isso permite uma redução do volume de gás natural, GN, adquirido no mercado e, por conseguinte, uma redução dos custos de produção.

\subsubsection{Planta de Carboquímicos}

Como efeito da adição do catalisador à mistura de carvões sobre a Planta de Carboquímicos, houve um aumento médio ao redor de 4,60 t/dia na produção de BTX (Benzeno, Tolueno, Xileno) e redução, na mesma proporção, da produção de alcatrão. Como consequência, houve um ganho financeiro potencial em função da maior margem de contribuição do BTX em comparação àquela do alcatrão.

\subsubsection{Limpeza dos "goosenecks" e tubos de ascensão}

Observou-se também durante o período dos testes industriais realizados na Bateria \# 1, uma redução na frequência de limpeza dos "goosenecks" e tubos de ascensão. Esse fato é extremamente relevante do ponto de vista da segurança do pessoal e do meio ambiente. A Figura 5 ilustra o interior do "gooseneck" de um forno da Bateria \# 1, com a utilização do catalisador, sem a presença de depósito de material (obstrução). 



Figura 5 - Vista do interior do "gooseneck" de um forno da Bateria \# 1 com a adição do catalisador, sem a presença de depósito de material (obstrução).

\subsection{Bateria de Fornos \# 4A, 4B e 5}

\subsubsection{Qualidade do coque}

A título de exemplificação, a Figura 6 apresenta uma comparação da qualidade do coque coletado na Rampa \# 3, não estabilizado, em termos do índice de resistência mecânica a frio, DI 150/15, com e sem a adição do catalisador, tomando-se como referência o Forno \# 153 da Baterias \# 5, bateria alta, mantendo-se o tempo total padronizado do processo de coqueificação, de 17 horas, visando à sua preservação, semelhante à Bateria \# 1. Conforme mencionado anteriormente, isso possibilita, alternativamente, a utilização de carvões de menor custo na mistura, sem prejuízos ao atendimento à especificação do coque para os Altos-Fornos, resultando numa redução dos custos com a aquisição dos carvões de coqueria.



Figura 6 - Comparação dos resultados de qualidade do coque, DI 150/15, coletado na Rampa \# 3, não estabilizado, do Forno \# 153 da Bateria \# 5.

* Contribuição técnica ao $44^{\circ}$ Seminário de Redução de Minério de Ferro e Matérias-primas, 15은 Simpósio Brasileiro de Minério de Ferro e $2^{\circ}$ Simpósio Brasileiro de Aglomeração de Minério de Ferro, 15 a 18 de setembro de 2014, Belo Horizonteg MG, Brasil. 


\subsubsection{Limpeza do sistema "PROven", "goosenecks", tubos de ascensão e empilhamento da Câmara de combustão}

À semelhança da Bateria \# 1, verificou-se também durante o período dos testes industriais com a adição do catalisador à mistura de carvões, realizados nas Baterias \# 4A, 4B e 5, baterias altas, uma melhoria na limpeza do sistema "PROven", "goosenecks", tubos de ascensão, empilhamento da câmara de combustão e paredes dos fornos. Como consequência, houve uma redução da frequência de limpeza, com impacto positivo do ponto de vista da segurança do pessoal, do meio ambiente e da produção - aumento da disponibilidade dos fornos.

A Figura 7 ilustra o aspecto de uma rolha nova, sem uso, e após o uso, com e sem a adição do catalisador.

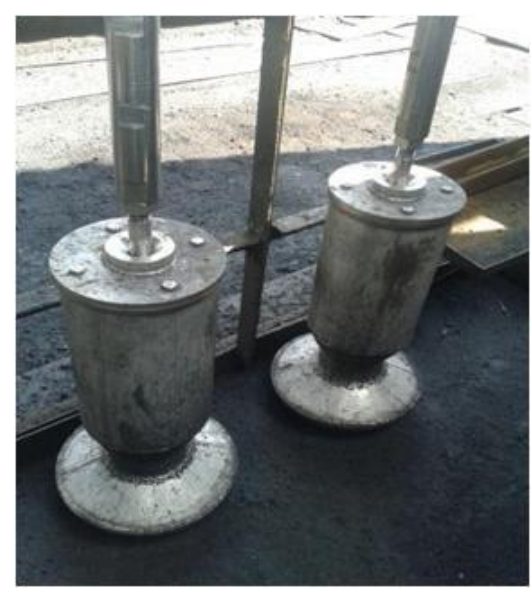

A) Rolha nova, sem uso.

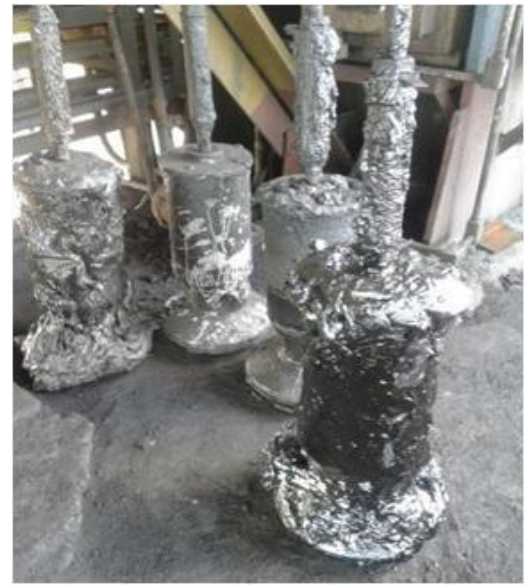

B) Rolha após uso, sem catalisador

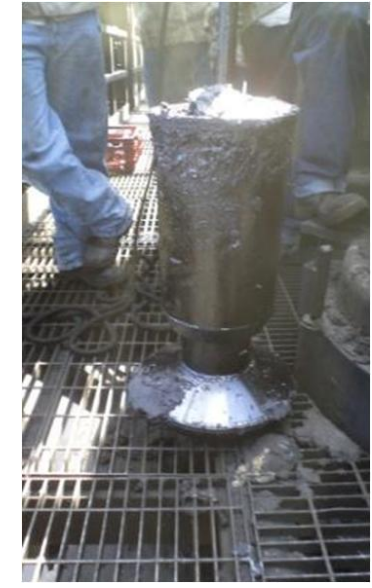

1. Rolha após uso, com catalisador.



Figura 7 - Aspecto da rolha nova, sem uso, e após o uso, com e sem a adição do catalisador, limpa e obstruída, respectivamente.

\subsubsection{Controle e ajuste automático da pressão dos fornos}

A melhoria da limpeza do sistema "PROven" concorreu também para a estabilidade do controle e ajuste automático da pressão dos fornos. A título de exemplificação, a Figura 8 ilustra a tela de controle e ajuste automático da pressão do Forno \# 162 da Bateria \# 5, antes e após a adição do catalisador. 


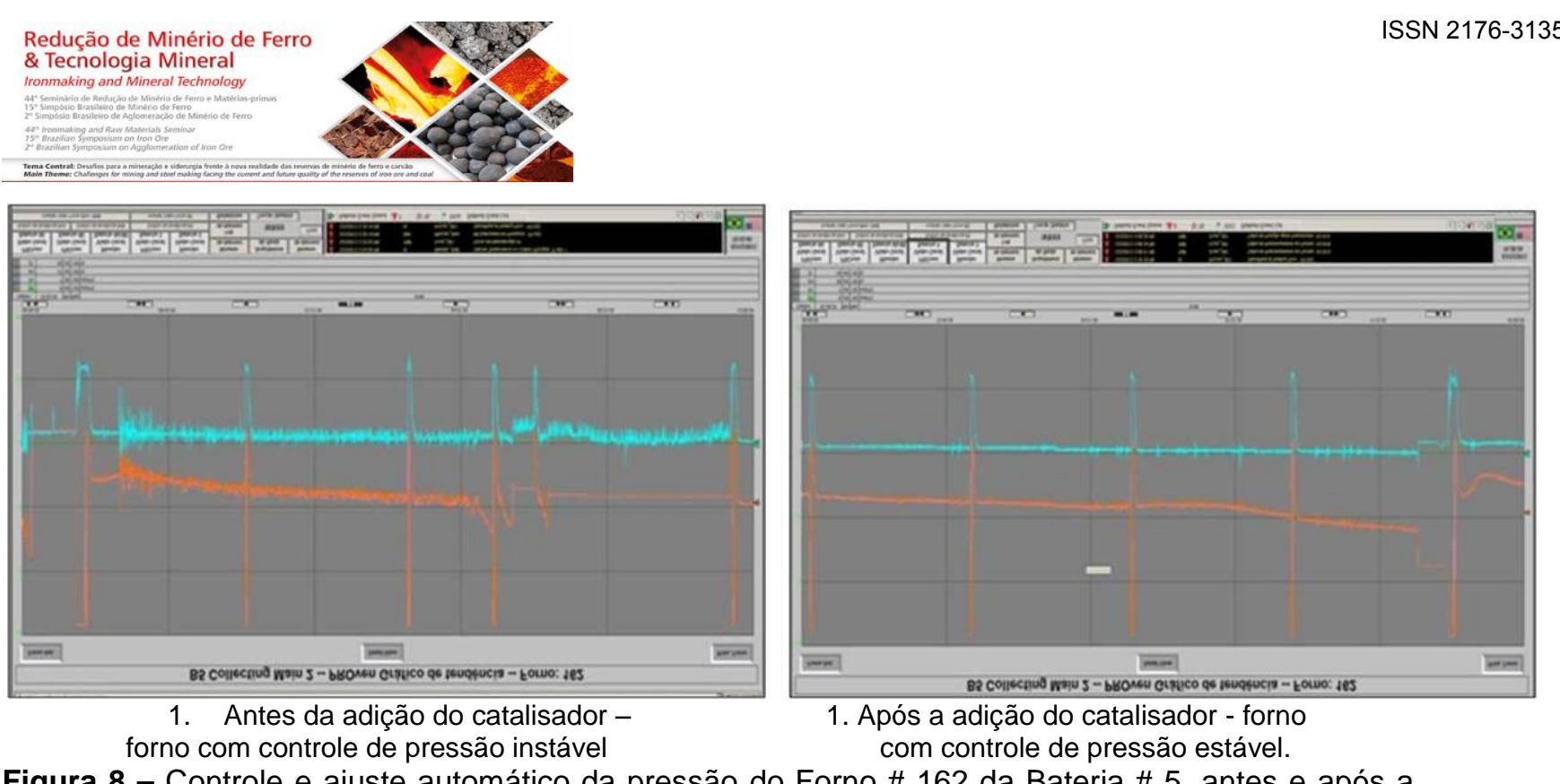

Figura 8 - Controle e ajuste automático da pressão do Forno \# 162 da Bateria \# 5, antes e após a adição do catalisador - controle automático.

\section{CONCLUSÃo}

Os resultados dos testes industriais realizados nas Baterias \# 1, 4A, 4B e 5 da CSN, com a adição do catalisador THERMACT à mistura de carvões, sinalizaram a viabilidade técnica e financeira da sua aplicação no processo da coqueria. Os principais benefícios decorrentes da adição do catalisador foram:

i) Aumento potencial da capacidade de produção de coque ao redor de $10 \%$ ou, alternativamente, melhoria da qualidade do coque - índice de resistência mecânica a frio, DI150/15, mantendo-se o tempo total padronizado do processo de coqueificação, visando à preservação das Baterias, ou ainda, a utilização de uma mistura de carvões de menor custo, sem prejuízos ao atendimento à especificação do coque para os Altos-Fornos.

ii) Aumento na geração e redução do consumo de gás de coqueria, GCO.

iii) Aumento na geração de BTX - Benzeno, Tolueno, Xileno, e redução proporcional da produção de alcatrão na Planta de Carboquímicos.

iv) Melhoria na limpeza do sistema "PROven", "goosenecks", tubos de ascensão, paredes dos fornos e empilhamento da câmara de combustão, com redução da frequência de limpeza, com impacto positivo dos pontos de vista da segurança do pessoal, do meio ambiente e da produção - aumento da disponibilidade dos fornos.

v) A melhoria da limpeza do sistema "PROven" concorreu também para a estabilidade do controle e ajuste automático da pressão dos fornos.

\section{REFERÊNCIA}

1 Abhitech Energycon Limited - Fuel Additives Manufacturer for Petroleum \& Solid Fuels.

Disponível em: www.abhitechenergycon.com. 